\title{
Chemical Fingerprinting of Polymers Using Electron Energy-Loss Spectroscopy
}

\author{
Ruchi Pal, Laure Bourgeois, Matthew Weyland, Arun K. Sikder, Kei Saito, Alison M. Funston,* \\ and Jayesh R. Bellare*
}

Cite This: ACS Omega 2021, 6, 23934-23942

Read Online

ABSTRACT: Electron energy-loss spectroscopy (EELS) is becoming an important tool in the characterization of polymeric materials. The sensitivity of EELS to changes in the chemical structure of polymeric materials dictates its applicability. In particular, it is important for compositional analysis to have reference spectra of pure components. Here, we report the spectra of the carbon K-edge of six polymers (polyethylene, polypropylene, polybutylene terephthalate, and polylactic acid) including copolymers (styrene acrylonitrile and acrylonitrile butadiene styrene), to be used as reference spectra for future EELS studies of polymers. We have successfully decomposed the carbon K-edge of each of the polymers and assigned the observed peaks to bonding transitions. The spectra have been acquired in standard experimental conditions, and

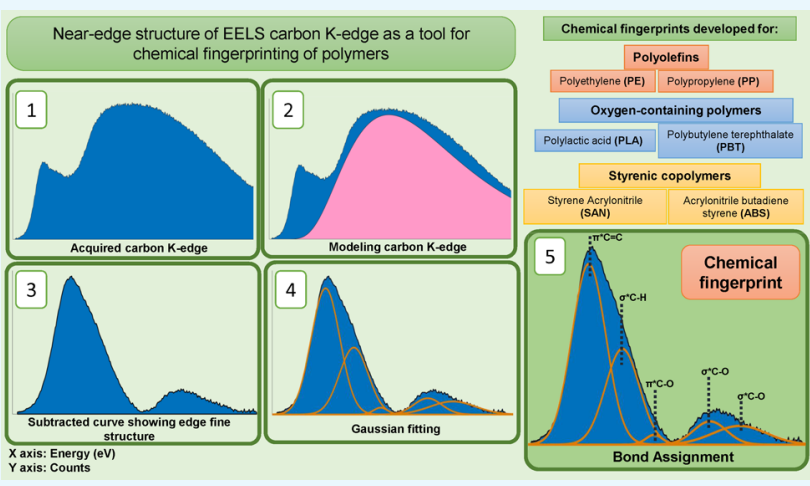
electron beam damage has been taken into account during establishment of spectral-structural relationships. We found that the more commonly available low-energy resolution spectrometers are adequate to chemically fingerprint linear saturated hydrocarbons such as PE, PP, and PLA. We have thus moved a step closer toward creating an atlas of polymer EELS spectra, which can be subsequently used for chemical bond mapping of polymeric materials with nanoscale spatial resolution.

\section{INTRODUCTION}

The applications of polymers are widespread in today's world. In order to meet the requirements of growing applications, chemical and morphological modifications are being carried out for next-generation polymeric materials. For example, inclusion of nanosized domains (e.g., in the form of fillers and reinforcements) in a polymeric matrix is a common method for producing multifunctional polymeric materials. In such applications, the study and understanding of nanostructured interfaces play a key role in determining, and consequently influencing, the material's properties.

Transmission electron microscopy (TEM) is a wellestablished tool to study materials at the nanoscale. Polymeric materials, however, present great challenges for TEM due to factors such as radiation damage and low contrast. One of the traditional solutions involves selectively incorporating heavy elements (such as ruthenium and osmium) in the sample, i.e., staining. Staining offers low versatility, unreliable quantitative information, and high toxicity, thereby posing difficulties in using this method for studying polymers in the TEM. Detailed characterization and imaging via TEM/electron energy-loss spectroscopy (EELS) provides a method for tackling this problem since EELS can reveal the rich electronic structure provided by polymers. ${ }^{1}$

EELS involves spectroscopic analysis of inelastic electronmatter interactions. When incoming electrons pass through a thin sample within a TEM, they undergo energy-loss. This energy-specific energy-loss is measured, resulting in an EELS spectrum. ${ }^{2}$ In particular, the energy-loss near-edge structure (ELNES) within the EELS spectrum contains peaks that are characteristic of the bond types present in the sample. Consequently, ELNES has been utilized to identify characteristic peaks in pure polymer EELS spectra, thereby producing chemical fingerprints of polymers. ${ }^{3}$ The complex covalent bonding of carbon atoms in polymers provides a particularly rich and detailed ELNES of the carbon K-edge. Investigations of carbon K-edge ELNES are therefore well-suited to study polymers. ${ }^{4}$ Additionally, as it is a TEM-based technique, EELS has potential subnanoscale spatial resolution, allowing potentially the mapping of different polymeric species at the nanoscale.

Received: June 4, 2021

Published: September 13, 2021

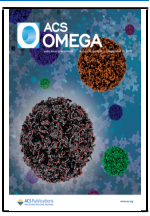


X-ray absorption spectroscopy (via NEXAFS; near-edge Xray absorption fine structure) has also been exploited as a fingerprinting tool for polymer analysis. ${ }^{6,7}$ The NEXAFS data in XAS is analogous to ELNES data in EELS, ${ }^{8}$ although it lacks the high spatial resolution of EELS, and requires synchrotron radiation. This synchrotron-based high-energy resolution technique is, however, a useful starting point for polymer identification using bond assignments.

Despite its advantages, EELS, as a characterization technique to study polymeric materials, remains largely under-utilized. One of the major reasons is the high beam sensitivity of polymeric materials. Polymers are prone to damage under an electron beam either by chain scission or cross-linking. However, standard experimental conditions such as a low electron dose, STEM (scanning transmission electron microscopy) mode, and cryo-TEM are employed widely to minimize beam damage. ${ }^{10}$ It then becomes more important to understand the spectral features due to beam damage on the EELS spectra under standard experimental conditions while minimizing beam damage. Despite this, beam damage is, however, still likely to be observed in any EEL spectra collected using relatively standard TEM/EELS instrumentation. This does not preclude the use of EELS as a characterization technique but rather requires full characterization of polymer spectra including identification of loss peaks, which result from radiation damage to the polymer itself, and accurate knowledge of experimental parameters. This will ensure greater applicability of EELS as a mapping technique for identification of polymers at the nanoscale.

The utility of a characterization technique such as EELS depends strongly on the sensitivity of EELS to the specific chemical structure of the polymer. The only fully analyzed polymer EELS carbon K-edge spectra to date are those of polystyrene (PS), ${ }^{11}$ polymethylmethacrylate (PMMA), ${ }^{12,13}$ polyethylene terephthalate (PET), ${ }^{14}$ polytetrafluoroethylene (PTFE), ${ }^{15}$ polyvinyl acetate (PVAc), ${ }^{3}$ and polycarbonate (PC). ${ }^{3}$ Therefore, a comprehensive atlas and catalog of the various polymers of importance and their corresponding carbon K-edge spectra with bond assignments are a prerequisite for the full exploitation of EELS as a polymer characterization and mapping tool.

Toward this goal, here, we report a set of spectra from six common polymers that represent a range of functionalities. Each reference spectrum reported here shows a unique spectral signature that can be used as a fingerprint for compositional analysis. The EEL spectra are collected using relatively standard and therefore accessible TEM/EELS instrumentation. As a consequence of this, chemical information obtained includes that related to beam damage, and spectral peaks resulting from beam damage are analyzed and reported. In this work, available information from the XAS/NEXAFS was utilized to corroborate the observations in our EELS results. We present EEL spectra of the polymers polyethylene (HDPE), polypropylene (PP), polybutylene terephthalate (PBT), polylactic acid (PLA), styrene acrylonitrile copolymer (SAN), and acrylonitrile butadiene styrene copolymer (ABS). This selection of polymers encompasses pure hydrocarbons (polyolefins) as well as oxygen-containing polymers and styrenic polymers. We conclude by discussing the core-loss spectra of styrenic copolymers SAN and ABS. We find that the same bonding transition occurs at different energies, as the polymer structure changes, due to a change in the local chemical environment. This leads to a range of energy values for any bonding transition. While this may lead to high probability of peak overlap, we find that detecting the presence of a heteroatom in an unknown polymer may be possible via the carbon K-edge ELNES. The energy resolution used could be reliably used to study linear saturated hydrocarbons, viz., PE, PP, and PLA. ${ }^{3}$ The reported spectra can potentially be employed to understand interfacial properties (such as interfacial width and interfacial composition) at the nanoscale in polymeric materials, ${ }^{16}$ where a change in bonding occurs over nanolength scales.

\section{RESULTS AND DISCUSSION}

We begin this section with the energy-loss spectra of simple hydrocarbon chain polymers such as the polyolefins PE and PP. Further, the effects of adding an oxygen-containing pendant group to the polymer backbone on the EELS carbon K-edge are discussed using the examples of oxygen-containing polymers such as PLA and PBT. Our results for the EELS chemical fingerprint of PET, which agree with previous EELS studies, ${ }^{14,21}$ are also mentioned here for comparison. Finally, the core-loss spectra of styrenic copolymers SAN and ABS, as well as their comparison to the pure PS spectrum, are discussed at the end of the section.

2.1. Polyolefins. The polyolefins polyethylene (highdensity) (HDPE) and polypropylene (PP) are fully saturated and are therefore expected to have relatively simple EEL spectra consisting of electronic transitions to empty $\pi^{*}$ and $\sigma^{*}$ orbitals. These two polymers differ structurally via the presence of a methyl $\left(-\mathrm{CH}_{3}\right)$ group on every alternating carbon.

EELS results of $\mathrm{PE}$ and $\mathrm{PP}$, showing the processed spectra decomposed into individual Gaussian peaks, are depicted in Figure $1 \mathrm{a}, \mathrm{b}$, and the energies of the transitions are reported in Table 1. Electronic states and electronic excitations of polyethylene have been previously studied by EELS, but its carbon core-loss region has not been explored. ${ }^{16,19}$ Three peaks are apparent for each of the polyolefins. The peak at

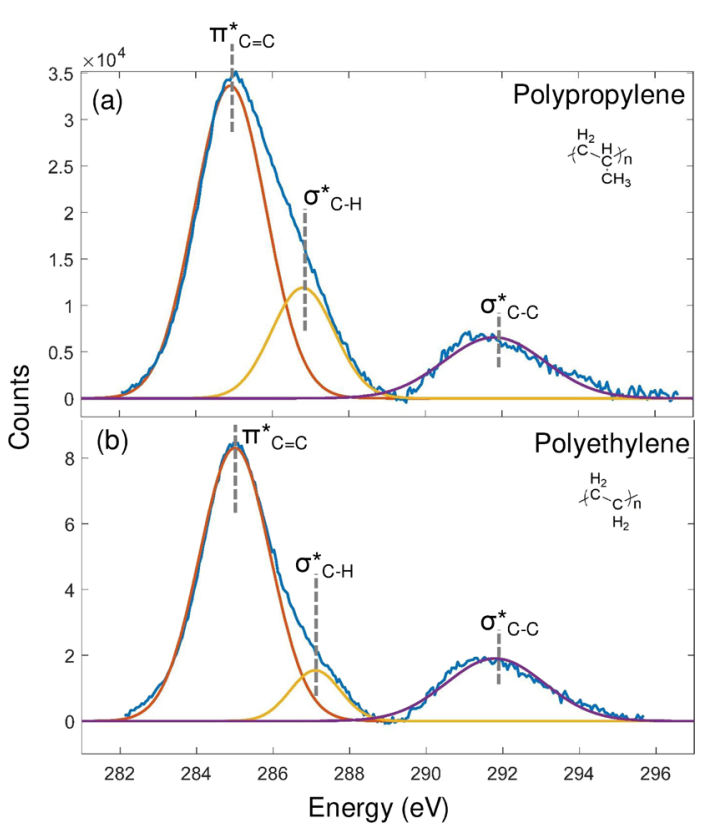

Figure 1. Carbon K-edge EELS spectra of (a) polypropylene (PP) and (b) polyethylene (PE), deconvolved into different Gaussian peaks using Tenailleau's model. 
Table 1. Bond Assignments for the Decomposed Carbon Edge of PE and PP

\begin{tabular}{cccc} 
peak & $\mathrm{PE}(\mathrm{eV})$ & $\mathrm{PP}(\mathrm{eV})$ & proposed assignments \\
1 & $285.0 \pm 0.1$ & $285.0 \pm 0.1$ & $\mathrm{C} 1 \mathrm{~s}-\pi^{*}{ }_{\mathrm{C}=\mathrm{C}}$ \\
2 & $286 \pm 1$ & $286.7 \pm 0.4$ & $\mathrm{C} 1 \mathrm{~s}-\sigma^{*}{ }_{\mathrm{C}-\mathrm{H}}$ \\
3 & $292.0 \pm 0.3$ & $292.1 \pm 0.6$ & $\mathrm{C} 1 \mathrm{~s}-\sigma^{*}{ }_{\mathrm{C}-\mathrm{C}}$ \\
\hline
\end{tabular}

$285.0 \mathrm{eV}$ in both the polymers is due to the $\mathrm{C} 1 \mathrm{~s}-\pi^{*} \mathrm{C}=\mathrm{C}$ transition. The unsaturated bonds are introduced as a result of electron beam damage during irradiation. ${ }^{16}$ The second peak occurs at $286 \pm 1$ and $286.7 \pm 0.4 \mathrm{eV}$ in $\mathrm{PE}$ and PP, respectively. Since the feature of $\mathrm{C} 1 \mathrm{~s}-\sigma^{*}{ }_{\mathrm{C}-\mathrm{H}}$ is expected to occur around this energy, $3,11,12,14$ the second peak in both the polyolefins is attributed to the $\mathrm{C} 1 \mathrm{~s}-\sigma^{*}{ }_{\mathrm{C}-\mathrm{H}}$ transition. The low intensity peak at around $292.0 \mathrm{eV}$, in both the polymers, is ascribed to the $\mathrm{C} 1 \mathrm{~s}-\sigma^{*}{ }_{\mathrm{C}-\mathrm{C}}$ transitions. This assignment agrees with the NEXAFS literature of HDPE and PP where the C $1 \mathrm{~s}-\sigma^{*}{ }_{\mathrm{C}-\mathrm{C}}$ transitions occur in the range of $291.8-293.1$ $\mathrm{eV}^{4,20}$

High-resolution NEXAFS spectra of both the polyolefins show a minor difference, within errors, in the peak energies of the same bonding transitions. There is only one report that indicates different NEXAFS spectra for PP when compared to $\mathrm{PE}$, which shows the presence of an additional shoulder in polypropylene NEXAFS spectra at $287.2 \mathrm{eV}^{4}$ due to the presence of methyl groups along the hydrocarbon chain. In the present study, an instrumental energy resolution of $1.2 \mathrm{eV}$ limited the detection of this minor difference between PP and HDPE. However, it is clear from the NEXAFS literature, ${ }^{4,20}$ as well as EELS results, that the additional methyl group has only a weak influence on the EELS spectra of the polyolefins.

Polyethylene is often cross-linked using radiation to improve its properties and be used in applications such as food packaging. However, the dose of radiation required to achieve cross-linking is far higher than that in an electron microscope. $^{21}$

At low radiation doses, PE degrades by chain scission. ${ }^{22}$ Thus, the degradation mechanism followed by PE under a TEM involves unsaturation and hydrogen liberation rather than cross-linking. ${ }^{16,19,21,22}$ Moreover, PP is prone to oxidative degradation due to the presence of tertiary carbon atoms. ${ }^{23}$ In the absence of oxygen, however, electron beam exposure causes scission and cross-linking to similar extents. ${ }^{23}$

Finally, in both the polyolefins, the $285 \mathrm{eV}$ peak, attributed to unsaturation, is a result of chain scission occurring during beam damage.

2.2. Oxygen-Containing Polymers. Polylactic acid (PLA) is an oxygen-containing polymer having an ester group within the main chain. Polybutylene terephthalate (PBT) has a relatively complex structure with both an aromatic group and a carbonyl group in the main chain. The presence of oxygen in PLA and PBT leads to an increase in the number of peaks when compared to a saturated polyolefin.

The carbon K-edge of PLA was decomposed into five peaks as displayed in Figure 2a. The energy-loss peak values obtained for each transition in PLA are shown in Table 2. In addition to the $\mathrm{C} 1 \mathrm{~s}-\pi^{*}{ }_{\mathrm{C}=\mathrm{C}}$ peak due to radiation damage, a loss peak was observed at $288.5 \mathrm{eV}$, which was assigned to the $\mathrm{C} 1 \mathrm{~s}-\pi^{*}{ }_{\mathrm{C}}=\mathrm{O}$ transition in accordance with the PLA NEXAFS literature. ${ }^{24,25}$ The peak at $286.8 \mathrm{eV}$ in PLA energy-loss spectra is ascribed to the $\mathrm{C} 1 \mathrm{~s}-\sigma^{*}{ }_{\mathrm{C}-\mathrm{H}}$ bonding transition since this transition is observed to be within the $286.7-287.2 \mathrm{eV}$ energy range for all

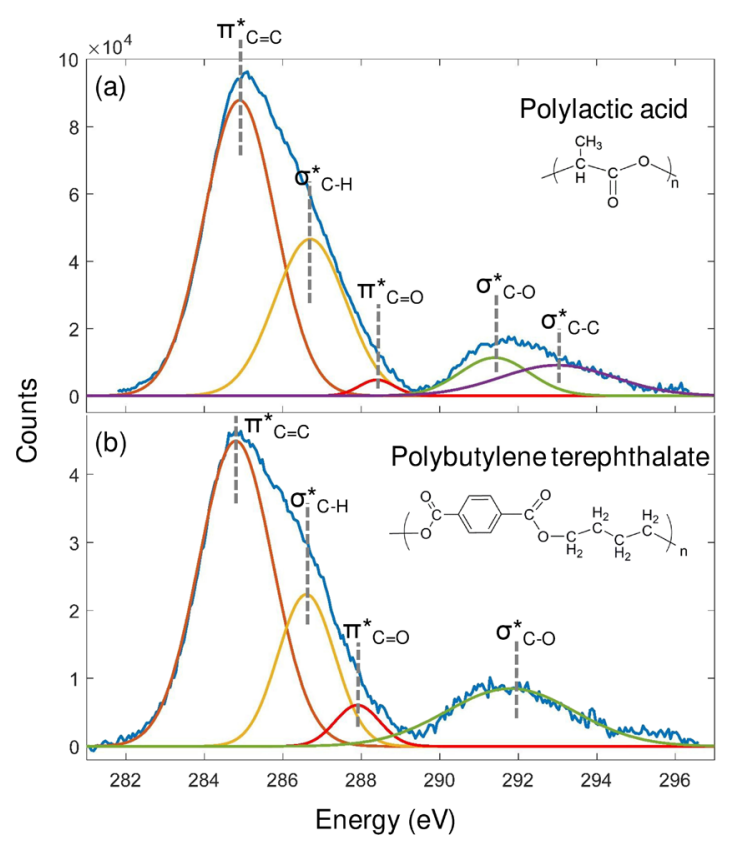

Figure 2. Carbon K-edge EELS spectra of (a) polylactic acid (PLA) and (b) polybutylene terephthalate (PBT), deconvolved into different Gaussian peaks using Tenailleau's model.

the homopolymers studied until now. ${ }^{3,11,12,14}$ The energy-loss peak at $289.6 \pm 0.5 \mathrm{eV}$ is assigned to the $\mathrm{C} 1 \mathrm{~s}-\sigma^{*}{ }_{\mathrm{C}-\mathrm{O}}$ transition in agreement with the reported peak energy of 290 $\mathrm{eV}$, for the same transition in NEXAFS. ${ }^{24,25}$ Similarly, the last discernible peak in PLA energy-loss spectra at $294 \pm 1 \mathrm{eV}$ is assigned to the $\mathrm{C} 1 \mathrm{~s}-\sigma^{*}{ }_{\mathrm{C}-\mathrm{C}}$ transition, very close (within error) to the reported value of $293.3 \mathrm{eV}$ by NEXAFS. ${ }^{24,25}$

The generally accepted radiation damage mechanism in PLA involves the formation of free radicals, which ultimately leads to chain scission. ${ }^{26,27}$ The major reaction intermediates are the radicals formed due to homolytic scission at the polymer backbone $\mathrm{C}-\mathrm{C}$ (reactants in Figure 3).

The unsaturation, as identified by the $285 \mathrm{eV}$ peak, is possibly due to the rearrangement of the free radicals (Figure 3).

For PBT (Figure 2b), peak decomposition and assignment are challenging due to the complexity of its chemical structure, despite the availability of the NEXAFS literature. ${ }^{28,29}$ The bond assignments of PBT were thus carried out using previously reported PBT NEXAFS data and EELS results of structurally similar PET. ${ }^{14}$ Our results of the PET carbon Kedge along with the bond assignments are largely consistent with the literature ${ }^{14}$ (for details, see the Supporting Information, Figure S2 and assignments). However, a deviation from reported peak energy was observed for the $\mathrm{C} 1 \mathrm{~s}-\sigma^{*}{ }_{\mathrm{C}-\mathrm{O}}$ transition in PET. This deviation, however, matches closely with the value of $291.4 \mathrm{eV}$, possibly corresponding to a special excitation of $\mathrm{C}=\mathrm{O}$, as a result of electron irradiation damage. ${ }^{14,30}$ Detailed bond assignment of PET is given in the Supporting Information. In the carbon K-edge spectrum of $\mathrm{PBT}$, the first and most prominent peak at $285 \mathrm{eV}$ is assigned to the $\mathrm{C} 1 \mathrm{~s}-\pi^{*}{ }_{\mathrm{C}=\mathrm{C}}$ transition in the phenyl ring. Similar to $\mathrm{PET}$, it is possible that the unsaturation occurring as a result of beam damage also contributes to the intensity of the $285 \mathrm{eV}$ peak in the PBT carbon K-edge. The second and third peaks at 286.8 and $288.2 \mathrm{eV}$, respectively, are assigned to the $\mathrm{C}$ $1 \mathrm{~s}-\sigma^{*}{ }_{\mathrm{C}-\mathrm{H}}$ and $\mathrm{C} 1 \mathrm{~s}-\pi^{*}{ }_{\mathrm{C}=\mathrm{O}}$ transitions, consistent with the 
Table 2. Bond Assignments for the Decomposed Carbon Edge of PLA, PBT, and PET

\begin{tabular}{|c|c|c|c|c|c|}
\hline \multirow[t]{2}{*}{ peak } & \multirow[t]{2}{*}{ PLA $(\mathrm{eV}$} & \multicolumn{2}{|c|}{ PET $(e V)$} & \multirow[t]{2}{*}{ PBT $(\mathrm{eV})$} & \multirow[t]{2}{*}{ proposed assignments } \\
\hline & & this work & literature $^{14}$ & & \\
\hline 1 & $285.0 \pm 0.1$ & $285.0 \pm 0.1$ & 285.0 & $285.0 \pm 0.1$ & $\mathrm{C} 1 \mathrm{~s}-\pi^{*}{ }_{\mathrm{C}=\mathrm{C}}$ \\
\hline 2 & $286.8 \pm 0.1$ & $286.9 \pm 0.2$ & 286.4 & $286.8 \pm 0.1$ & $\mathrm{C} 1 \mathrm{~s}-\sigma^{*}{ }_{\mathrm{C}-\mathrm{H}}$ \\
\hline 3 & $288.5 \pm 0.1$ & $288.5 \pm 0.3$ & 288.7 & $288.2 \pm 0.2$ & $\mathrm{C} 1 \mathrm{~s}-\pi^{*}{ }_{\mathrm{C}=\mathrm{O}}$ \\
\hline 4 & $289.6 \pm 0.5$ & $291.9 \pm 0.1^{a}$ & 293.3 & $292.2 \pm 0.3$ & $\mathrm{C} 1 \mathrm{~s}-\sigma_{\mathrm{C}-\mathrm{O}}^{*}$ \\
\hline 5 & $294 \pm 1$ & $297.0 \pm 0.6$ & 296.7 & $b$ & $\mathrm{C} 1 \mathrm{~s}-\sigma^{*}{ }_{\mathrm{C}-\mathrm{C}}$ \\
\hline 6 & $b$ & $302.5 \pm 0.9$ & 302.1 & $b$ & $\mathrm{C} 1 \mathrm{~s}-\sigma_{\mathrm{C}=\mathrm{O}}^{*}$ \\
\hline 7 & $b$ & $303.6 \pm 0.2$ & 304.1 & $b$ & $\mathrm{C} 1 \mathrm{~s}-\sigma_{\mathrm{C}=\mathrm{C}}^{*}$ \\
\hline
\end{tabular}

${ }^{a}$ This peak was due to electron beam degradation and corresponds to a special excitation of $\mathrm{C}=\mathrm{O}$ transition. ${ }^{b}$ The data was too noisy within the expected energy range to be able to extract peaks for these transitions.

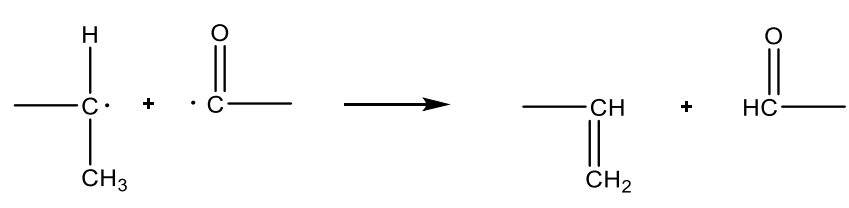

Figure 3. Proposed rearrangement of free radicals formed during radiation exposure to form carbon-carbon double bonds in PLA.

PET EELS data ${ }^{14}$ and the PBT NEXAFS literature. ${ }^{4,31}$ The broad peak at $292.2 \mathrm{eV}$ is ascribed to the $\mathrm{C} 1 \mathrm{~s}-\sigma^{*} \mathrm{C}-\mathrm{O}$ transition also supported by PET EELS results. ${ }^{14}$ However, the chemical complexity of PBT and the limitations of energy resolution suggest that there are potential signals from other transitions that affect this peak. Concurrent with PBT NEXAFS results, ${ }^{28}$ our findings suggest that the broad peak at $292.2 \mathrm{eV}$ may have signals from the $\mathrm{C} 1 \mathrm{~s}-\pi^{*}{ }_{\mathrm{C}=\mathrm{C}}$ transition of the phenyl ring as well.

Not surprisingly, as the structure of a polymer becomes increasingly complex, the limits of instrumental energy resolution decrease the accuracy of the chemical fingerprint of the polymer. ${ }^{9}$ With a highly complex chemical structure, limited energy resolution, and additional conjugation effects produced by the presence of phenyl groups, the assignment of spectral-structural relationships in PBT was complicated.

2.3. Styrenic Polymers and Copolymers. Polymerization of styrene results in the homopolymer polystyrene. Addition of the monomer acrylonitrile to styrene polymerization forms the copolymer SAN. Inclusion of a third monomer, butadiene, to the structure produces the copolymer ABS. However, ABS is essentially composed of a matrix of SAN having polybutadiene (PB) rubber particles cross-linked with SAN.

The EELS spectra of the carbon K-edge of PS have been reported previously, and our results are consistent with these (see the Supporting Information, Figure S1 and assignments). ${ }^{11}$ The EELS carbon K-edges of PS (Figure S1), SAN (Figure 4,ba), and ABS (Figure 4c) show an intense feature at $285 \mathrm{eV}$, a signature of the $\pi^{*}$ resonance in the phenyl pendant group. ${ }^{6,11,28,31}$ Small differences in the chemical structure of these materials lead to the overlap of signals from different bonds, making the spectral interpretation complex. In order to improve the integrity of the peak assignment for complex polymeric structures, the SAN carbon edge was also acquired at high-energy resolution (Figure $4 \mathrm{~b}$ ). Well-separated peaks can be seen in Figure 4b, although the data is relatively noisy. This noise may have come from an excessively thick sample under an $80 \mathrm{kV}$ electron beam or from instrument/power issues that could not be definitively determined. We note that after processing the low-energy

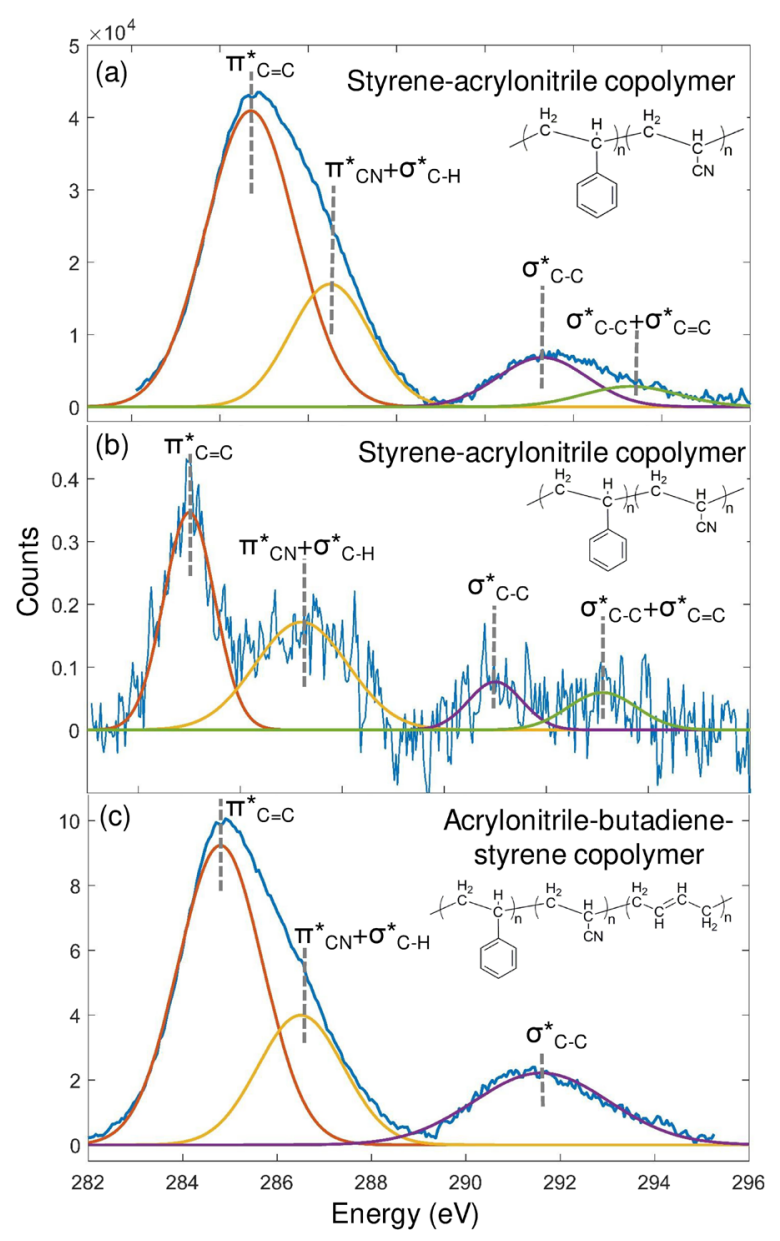

Figure 4. Carbon K-edge EELS spectra of the styrene acrylonitrile copolymer (SAN) at (a) low-energy resolution and (b) high-energy resolution; (c) carbon K-edge EELS spectra of the acrylonitrile butadiene styrene copolymer (ABS). All the spectra have been deconvolved into different Gaussian peaks using Tenailleau's model.

resolution data, the peak energy values obtained match closely with the high-resolution values; indeed, these are within the experimental error (shown in Table 3).

For PS, SAN, and ABS, a peak at around $287 \mathrm{eV}$ is present, although these are due to different structural features of the polymers. For PS, the peak at $287.1 \mathrm{eV}$ is due to the $\mathrm{C}$ $1 \mathrm{~s}-\sigma^{*}{ }_{\mathrm{C}-\mathrm{H}}$ transition in agreement with the previously reported value of $287.4 \mathrm{eV} .{ }^{11}$ However, in SAN and ABS, the peaks around $287 \mathrm{eV}$ are considered to be due to a combination of signals from the $\mathrm{C} 1 \mathrm{~s}-\pi^{*}{ }_{\mathrm{C}=\mathrm{N}}$ and the $\mathrm{C} 1 \mathrm{~s}-\sigma^{*}{ }_{\mathrm{C}-\mathrm{H}}$ transitions. This assignment is based on the EELS results of $\mathrm{PS}^{11}$ (see 
Table 3. Bond Assignments for the Decomposed Carbon Edge of the SAN Copolymer (High- and Low-Energy Resolution), ABS, and PS

\begin{tabular}{|c|c|c|c|c|c|c|}
\hline \multirow[t]{2}{*}{ peak } & \multicolumn{2}{|c|}{ SAN $(e V)$} & \multirow[t]{2}{*}{$\mathrm{ABS}(\mathrm{eV})$} & \multicolumn{2}{|c|}{ PS $(e V)$} & \multirow[t]{2}{*}{ proposed assignments $^{a}$} \\
\hline & low res. & high res. ${ }^{b}$ & & this work & literature $^{11}$ & \\
\hline 1 & $285.0 \pm 0.1$ & 285.0 & $285.0 \pm 0.1$ & $285.0 \pm 0.1$ & 285.0 & $\mathrm{C} 1 \mathrm{~s}-\pi^{*} \mathrm{C}=\mathrm{C}$ \\
\hline 2 & $286.9 \pm 0.1$ & 287.2 & $286.8 \pm 0.1$ & $287.1 \pm 0.5$ & 287.4 & $\mathrm{C} 1 \mathrm{~s}-\sigma_{\mathrm{C}-\mathrm{H}}^{*}\left[+\mathrm{C} 1 \mathrm{~s}-\pi_{\mathrm{C}=\mathrm{N}}^{*}\right]$ \\
\hline 3 & $291.8 \pm 0.3$ & 291.0 & $291.8 \pm 0.2$ & $291.6 \pm 0.2$ & 293.3 & $\mathrm{C} 1 \mathrm{~s}-\sigma^{*}{ }_{\mathrm{C}-\mathrm{C}}$ \\
\hline 4 & $295 \pm 1$ & 293.1 & & $302.4 \pm 0.4$ & 303.0 & $\mathrm{C} 1 \mathrm{~s}-\sigma_{\mathrm{C}=\mathrm{C}}^{*}\left[+\mathrm{C} 1 \mathrm{~s}-\sigma_{\mathrm{C}-\mathrm{C}}^{*}\right]$ \\
\hline
\end{tabular}

${ }^{a}$ Square brackets indicate an additional contribution from other transitions for copolymers SAN and ABS. ${ }^{b}$ High-resolution data is reported from only one sample region; therefore, no error is reported.

Table 4. Binding Energies along with Their Bond Assignments for All Polymers under Consideration ${ }^{a}$

\begin{tabular}{|c|c|c|c|c|c|c|c|c|c|c|c|c|}
\hline S.No. & $\begin{array}{l}\text { Proposed } \\
\text { assignments }\end{array}$ & $\mathrm{PE}$ & PP & PLA & PBT & SAN & ABS & PS $11 \mathrm{~b}$ & PET14 b & PMMA $^{12 b}$ & $\mathrm{PVAc}^{3} \mathrm{c}$ & $\mathrm{PC}^{3} \mathrm{c}$ \\
\hline \multirow[t]{2}{*}{1.} & \multirow[t]{2}{*}{ C $1 \mathrm{~s}-\pi^{*} \mathrm{C}=\mathrm{C}$} & \multirow[t]{2}{*}{$285.0 \pm 0.1$} & \multirow[t]{2}{*}{$285.0 \pm 0.1$} & \multirow[t]{2}{*}{$285.0 \pm 0.1$} & \multirow[t]{2}{*}{$285.0 \pm 0.1$} & $285.0 \pm 0.1$ & \multirow[t]{2}{*}{$285.0 \pm 0.1$} & $285.0 \pm 0.1$ & $285.0 \pm 0.1$ & $285.0 \pm 0.1$ & $285.0 \pm 0.1$ & $285.0 \pm 0.1$ \\
\hline & & & & & & 285.0 & & 285.0 & 285.0 & 285.0 & $285.0 \pm 0.2$ & $285.0 \pm 0.1$ \\
\hline \multirow[t]{2}{*}{2.} & \multirow[t]{2}{*}{ C $1 \mathrm{~s}-\sigma^{*} \mathrm{C}-\mathrm{H}$} & \multirow[t]{2}{*}{$286 \pm 1$} & \multirow[t]{2}{*}{$286.7 \pm 0.4$} & \multirow[t]{2}{*}{$286.8 \pm 0.1$} & \multirow[t]{2}{*}{$286.8 \pm 0.1$} & & & $287.1 \pm 0.5$ & $286.9 \pm 0.2$ & $287.1 \pm 0.1$ & $286.6 \pm 0.4$ & $286.9 \pm 0.1$ \\
\hline & & & & & & & & 287.4 & 286.4 & 287.2-287.5 & $286.8 \pm 0.1$ & $286.9 \pm 0.1$ \\
\hline \multirow[t]{2}{*}{3.} & \multirow[t]{2}{*}{ C $1 \mathrm{~s}-\pi^{*} \mathrm{C}=\mathrm{N}$} & & & & & $286.9 \pm 0.1^{\mathrm{d}}$ & \multirow[t]{2}{*}{$286.8 \pm 0.1^{\mathrm{d}}$} & & & & & \\
\hline & & & & & & $287.2^{d}$ & & & & & & \\
\hline \multirow[t]{2}{*}{4.} & \multirow[t]{2}{*}{$\mathrm{C} 1 \mathrm{~s}-\pi^{*} \mathrm{C}=0$} & & & \multirow[t]{2}{*}{$288.5 \pm 0.1$} & \multirow[t]{2}{*}{$288.2 \pm 0.2$} & & & & $288.5 \pm 0.3$ & $288.4 \pm 0.1$ & $287.6 \pm 0.6$ & $290.4 \pm 0.1$ \\
\hline & & & & & & & & & 288.7 & 288.2-288.6 & $287.6 \pm 0.2$ & $290.3 \pm 0.2$ \\
\hline \multirow[t]{2}{*}{5.} & \multirow[t]{2}{*}{ C $1 \mathrm{~s}-\sigma^{*} \mathrm{C}-\mathrm{O}$} & & & \multirow[t]{2}{*}{$289.6 \pm 0.5$} & \multirow[t]{2}{*}{$292.2 \pm 0.3$} & & & & $291.9 \pm 0.1$ & $291.1 \pm 0.1$ & $292.3 \pm 0.3$ & $292.0 \pm 0.3$ \\
\hline & & & & & & & & & 293.3 & $290.6-291.0$ & $291.0 \pm 0.2$ & $292.0 \pm 0.2$ \\
\hline \multirow[t]{2}{*}{6.} & \multirow[t]{2}{*}{ C 1s- $\sigma^{*} \mathrm{C}-\mathrm{C}$} & \multirow[t]{2}{*}{$292.0 \pm 0.3$} & \multirow[t]{2}{*}{$292.1 \pm 0.6$} & \multirow[t]{2}{*}{$294 \pm 1$} & & $291.8 \pm 0.3$ & \multirow[t]{2}{*}{$291.8 \pm 0.2$} & $291.6 \pm 0.2$ & $297.0 \pm 0.6$ & $292.6 \pm 0.1$ & & $295 \pm 1$ \\
\hline & & & & & & 291.0 & & 293.3 & 296.7 & 292.0-293.0 & & $295 \pm 1$ \\
\hline \multirow[t]{2}{*}{7.} & \multirow[t]{2}{*}{ C $1 \mathrm{~s}-\sigma^{*} \mathrm{C}=0$} & & & & & & & & $302.5 \pm 0.9$ & & & \multirow[t]{2}{*}{$304 \pm 1$} \\
\hline & & & & & & & & & 302.1 & & & \\
\hline \multirow[t]{2}{*}{8.} & C $1 \mathrm{~s}-\sigma^{*} \mathrm{C}=\mathrm{C}$ & & & & & $295 \pm 1^{d}$ & & $302.4 \pm 0.4$ & $303.6 \pm 0.2$ & & & \\
\hline & & & & & & 293.1 ${ }^{d}$ & & 303.0 & 304.1 & & & \\
\hline
\end{tabular}

${ }^{a}$ Shaded cells indicate the original data presented within this manuscript. White cells indicate data available in the literature. ${ }^{3,11-14}$ Values in bold, in the original data and from the literature, were obtained from high-energy resolution $(0.5-0.7 \mathrm{eV})$ EELS data. ${ }^{b}$ The shaded cells indicate the original data presented here, which are largely consistent with the literature. ${ }^{11-14}$ Details are provided in the Supporting Information. ${ }^{c}$ Authors' previously published work. ${ }^{3}{ }^{2}$ These peaks are a combination of signals from multiple bonding transitions.

Supporting Information) and NEXAFS results of PAN, which show the $\pi^{*}$ signature of $-\mathrm{CN}$ at around $286.8 \mathrm{eV}$. 6,32

In both the copolymers, the peaks at $291.0 \mathrm{eV}$ in SAN and $291.8 \mathrm{eV}$ in $\mathrm{ABS}$ are ascribed to the $\mathrm{C} 1 \mathrm{~s}-\sigma^{*}{ }_{\mathrm{C}-\mathrm{C}}$ transition from the PS units. The feature at higher energies in SAN $(293.1-295 \mathrm{eV})$ is a broad peak due to the $\sigma^{*}$ transition. This broad peak may have a contribution from both the $\mathrm{C}$ $1 \mathrm{~s}-\sigma^{*}{ }_{\mathrm{C}-\mathrm{C}}$ and the $\mathrm{C} 1 \mathrm{~s}-\sigma^{*}{ }_{\mathrm{C}=\mathrm{C}}$ transitions. ${ }^{32}$

The electron beam damage mechanism is established for PS but not for SAN and ABS. When considering the radiation chemistry of the copolymers SAN and ABS, we must take into account the radiation damage mechanisms of their respective homopolymers. PS damages under an electron beam by crosslinking via the phenyl ring. ${ }^{11}$ The damage effect will be seen in spectral features related to $\mathrm{C}-\mathrm{C}$ and $\mathrm{C}=\mathrm{C} \sigma^{*}$ transition values. Furthermore, PAN and $\mathrm{PB}$ are damaged under $\gamma$ radiation, within vacuum, predominantly by cross-linking. ${ }^{33}$ Acrylonitrile and butadiene units are more radiation-sensitive than styrene units. ${ }^{33}$ However, styrene-containing polymers, such as SAN and ABS, are known to undergo damage under $\gamma$ radiation via the formation of phenyl radicals. Essentially, the styrene units protect other susceptible units (such as AN) through electron transfer. ${ }^{33}$ The electron beam damage mechanism of the copolymers, SAN and ABS, would then proceed in a similar manner to that of polystyrene electron beam degradation. The damage signature in the EELS spectra of $\mathrm{ABS}$ and SAN would be similar to PS and is, in fact, exhibited by the presence of a peak at around $291 \mathrm{eV}$. The bond assignments for each of the polymers are tabulated in Table 4, including those obtained from high-resolution data. The table also summarizes, for comparison, the chemical fingerprints previously published for PS, ${ }^{11} \mathrm{PET}^{12}{ }^{12} \mathrm{PMMA}^{14}$ PVAc, ${ }^{3}$ and PC. ${ }^{3}$ This table can be used to identify signature peaks of a polymer or to differentiate peaks within a group of polymers. Electron energy-loss spectroscopy-spectrum imaging (EELS-SI) may potentially be employed by selecting a "differentiating energy range" to carry out chemical bond mapping in multiphase materials.

It can be seen from the table that the same bonding transition occurs at different energies, as the polymer structure changes, due to a change in the local chemical environment. Examples are the transitions related to carbon-oxygen bonds. The $\mathrm{C} 1 \mathrm{~s}-\sigma_{\mathrm{C}-\mathrm{O}}^{*}$ transition occurs in all the oxygen-containing polymers (PLA, PBT, PET, PMMA, PVAc, and PC). In PMMA and PVAc, the carbonyl group is isolated in the side chain of the polymer, while in other polymers, the carbonyl group is either present within the polymer backbone chain or is directly attached to it. The result is a change in binding energy of the $\mathrm{C} 1 \mathrm{~s}-\sigma^{*}{ }_{\mathrm{C}-\mathrm{O}}$ transition from a lower value of around $291.0 \mathrm{eV}$ in PVAc to higher values of $293.7 \mathrm{eV}$ in PBT.

Although the peak energy for a given bonding transition can spread over a wide range, the detection of a heteroatom in an unknown polymer may be possible using the carbon K-edge fine structure. As is evident from Table 4, the range that covers 
energies for the $\mathrm{C} 1 \mathrm{~s}-\sigma^{*}{ }_{\mathrm{C}-\mathrm{O}}$ bonding transition is $291.0-$ $293.7 \mathrm{eV}$. However, the energy range for the $\mathrm{C} 1 \mathrm{~s}-\pi^{*}{ }_{\mathrm{C}=\mathrm{N}}$ transition is $286.8-287.2 \mathrm{eV}$. The signature peaks of each of the heteroatoms (nitrogen and oxygen) are, therefore, in different energy ranges, making it possible to clearly identify the heteroatom. Nevertheless, a complete EELS chemical fingerprint of the polymer, including the low-loss region as well as ELNES of the core-loss edges of all the elements present, is likely to be required while differentiating among polymers.

An example where energy for a given transition is relatively invariant across a number of polymer structural classes is the $C$ $1 \mathrm{~s}-\sigma_{\mathrm{C}-\mathrm{H}}^{*}$ transition. This bond occurs in all the hydrocarbons, but its value mostly stays within a narrow range of 286.8$287.5 \mathrm{eV}$ for the polymer classes of polyolefins, PS, and oxygen-containing polymers (PLA, PMMA, PET, PBT, PVAc, and PC). However, for nitrogen-containing copolymers (SAN and $\mathrm{ABS}$ ), the binding energy value of the $\mathrm{C} 1 \mathrm{~s}-\sigma^{*} \mathrm{C}-\mathrm{H}$ transition is within the energy range of $286.8-291.8 \mathrm{eV}$.

Finally and as mentioned earlier, the $\pi^{*}$ transitions occur at lower energies than $\sigma^{*}$ transitions. It is noticeable from Table 4 that the error values are higher for peaks corresponding to $\sigma^{*}$ transitions as compared to the errors on $\pi^{*}$ transition-related peaks. This is due to the broad nature of peaks for $\sigma^{*}$ transitions in contrast to the narrow peaks for $\pi^{*}$ transitions.

\section{CONCLUSIONS}

We have presented the energy-loss spectra at the carbon Kedge for six polymers and copolymers. The carbon K-edge fine structure has been assigned to the different bonding transitions. The unique combinations of peak energy values are the chemical fingerprints for these polymers. Table 4 shows the bond assignments for each of the polymers taken into consideration, including those obtained from high-energy resolution EELS data as well as those of PET, PS, PMMA (reproduced from the literature), PVAc, and PC. The binding energy values from EELS results match closely to the highresolution NEXAFS values taken from the literature. In fact, results from the more commonly available low-energy resolution EELS instruments are adequate to study linear saturated polymers such as PE, PP, and PLA. This role of energy resolution in investigating polymers supports our previously published chemical fingerprint of PVAc. ${ }^{3}$ By documenting EELS spectral changes, we hope to provide an initial "catalog" of spectra for users of EELS spectroscopy tools, which have been collected under relatively standard EELS conditions.

The limitation in the applicability of EELS, however, is that all major changes in the spectra occur in a narrow energy range between 285 and about $295 \mathrm{eV}$ due to which there is a high possibility of overlap of contributions of different chemical moieties. Nevertheless, the latest TEM/EELS systems having electron guns with improved energy resolution, ranging from $0.3 \mathrm{eV}$ for a cold FEG to $25-100 \mathrm{meV}$ for monochromated instruments, may greatly help in overcoming this limitation. Additionally, more sensitive detectors may allow the samples to be characterized with much lower doses and hence before beam damage takes place.

This study has shown that small but measurable differences in energy and shape exist in the core excitation spectra of the polymers with similar elemental composition. The peak energy values can be used when employing electron energy-loss spectroscopy spectrum imaging (EELS-SI) to spatially differentiate between polymers of similar elemental make-up. By allowing mapping of phase segregation, these reference spectra not only provide better visualization of morphology in polymeric materials but also give chemical knowledge about the various domains. These can be highly beneficial to studying polymeric materials with strong structure-property correlation. Complete chemical fingerprints of the polymers can be developed by including the edge fine structure of all the elements present in the polymer along with these carbon Kedge spectra. Accurate energy scales and good energy resolution will be required.

Complementary theoretical calculations, not shown here, should also assist with the full assignment of the loss peaks observed in the fine structure. The complete chemical fingerprints will make it possible to identify unknown polymeric materials.

\section{EXPERIMENTAL SECTION}

4.1. Materials. HDPE (CC254-00900), PP (5707N), and PBT pellets were supplied by SABIC, India. Polylactic acid (4060D) was obtained from Natureworks, Australia. Copolymer SAN pellets were procured from Bayer, India, and ABS (SP 200) pellets were bought from Polimaxx, Australia. These were all used as received. Details of polymer characteristics are provided in the Supporting Information.

4.2. Sample Preparation. The polymers were cut into thin slices using an EM UC-S ultramicrotome (Leica) at room temperature. The sections were around $50 \mathrm{~nm}$-thick. Molded sheets of PE, PP, PLA, SAN and ABS, and PBT pellets were used for ultramicrotomy. An ultrasonic diamond knife was employed for room temperature ultramicrotomy of PP molded sheets. The polymer sections were adequately thin for EELS analysis since their thickness was measured to be in the range of $0.5-0.9$ inelastic mean free path. The sections were deposited on 300 mesh lacey carbon grids, procured from EMResolutions, and subsequently air-dried.

4.3. Energy-Loss Spectral Acquisition. EELS spectra were analyzed from five to eight different regions of each sample. To minimize radiation damage during acquisition of EEL spectra, ${ }^{16,17}$ a cold stage (Gatan liquid nitrogen holder 636DT) was used in the TEM. The cold stage helped maintain a temperature of $-180^{\circ} \mathrm{C}$ during the spectral acquisition. The sample was loaded on a cryo holder at room temperature, and after inserting it in the TEM, the temperature was reduced to $-180^{\circ} \mathrm{C}$.

A JEOL JEM 2100F, a $200 \mathrm{kV}$ field emission gun TEM, equipped with a Gatan Enfina EELS spectrometer, was used in TEM mode to acquire the spectra. The energy resolution was measured to be $1.2 \mathrm{eV}$. The spectra were recorded from an area of approximately $5.02 \times 10^{8} \AA^{2}$. The data for PS, PET, and PMMA were acquired at $200 \mathrm{kV}$ accelerating voltage, and for the remaining polymers, the accelerating voltage was $120 \mathrm{kV}$ to improve the energy resolution to $0.9 \mathrm{eV}$. An energy dispersion of $0.05 \mathrm{eV} /$ channel with an acquisition time of $0.5 \mathrm{~s} /$ frame, summed over 2 frames, was used along with a current density of $510 \pm 5 \mathrm{pA} / \mathrm{cm}^{2}$ to obtain a carbon K-edge. A lower acquisition time of $0.05 \mathrm{~s} /$ frame, summed over 10 frames, was used to get the low-loss spectra keeping energy resolution and current density the same. The electron dose was estimated to be approximately $310 \mathrm{e}^{-} \AA^{-2}$. High-energy resolution (0.51 $\mathrm{eV}$ ) data was acquired using a dual aberration-corrected FEI TITAN $^{3}$ 80-300, operated at $80 \mathrm{kV}$, equipped with a Gatan Tridium $863 \mathrm{P}$ image filter. The carbon K-edge was obtained through STEM mode at an energy dispersion of $0.03 \mathrm{eV} /$ 
channel with an exposure time of $2 \mathrm{~s}$ and a total integration time of $40 \mathrm{~s}$. To acquire the low-loss spectra from the same region, the exposure time was reduced to $0.1 \mathrm{~s}$. The electron dose was estimated to be approximately $50 \mathrm{e}^{-} \AA^{-2}$. In general, the low-loss spectra are acquired to allow removal of plural scattering effects by Fourier ratio deconvolution. The energy resolution is measured from the full width at half maximum (FWHM) of the zero-loss peak over a hole in the substrate.

All the spectra exhibit a strong feature at $285 \mathrm{eV}$ due to the $\mathrm{C} 1 \mathrm{~s}-\pi^{*}{ }_{\mathrm{C}=\mathrm{C}}$ electronic transition, irrespective of the presence of carbon-carbon double bonds in the polymer structure and even for the shortest exposure times. This is ascribed in the literature $^{9,10}$ to beam damage, consistent with other EEL spectra of polymers. Electron beam damage will be discussed for each polymer in subsequent sections. Surface adsorption of contaminants such as hydrocarbons from the environment or the vacuum system may also contribute to this signal. This peak has been used to calibrate each carbon K-edge spectrum.

4.4. Spectral Processing. The spectral processing methodology that has been earlier used for studying the ELNES of PC and PVAc was used here. ${ }^{3}$ Several post processing steps were carried out to the recorded carbon Kedge. A power law model was applied to perform background subtraction, which was followed by Fourier ratio deconvolution (to remove thickness effects), and finally, prepeak calibration of the carbon K-edge to $285 \mathrm{eV}$ was carried out.

The resulting spectra were used for extracting the edge fine structure. The carbon K-edge was simulated using the empirical model proposed by Tenailleau ${ }^{18}$ as given in eq 1

$$
I(E)=I_{\mathrm{m}}\left(\frac{E_{\mathrm{m}}}{E}\right)^{-r} \exp \left(\frac{r\left(E_{\mathrm{m}}-E_{\mathrm{k}}\right)\left(E-E_{\mathrm{m}}\right)}{E_{\mathrm{m}}\left(E-E_{\mathrm{k}}\right)}\right)
$$

where $I_{\mathrm{m}}$ and $E_{\mathrm{m}}$ are the coordinates of the maximum, $E_{\mathrm{k}}$ represents the starting point of the simulated curve and is generally dependent on the ionization potential, and $r$ is a variable that depends on the width of the model. The simulated carbon K-edge absorption peak was subtracted from the experimental spectrum to give the energy-loss near edge structure (ELNES). This model has been previously used for EELS spectral decomposition of PS, ${ }^{11}$ PMMA, ${ }^{12}$ PC, ${ }^{3}$ PVAc, ${ }^{3}$ and PET. ${ }^{14}$

The model given by eq 1 was fit to the experimental data within a specific energy range. The energy range for model fitting varies according to the structure of the polymer since it is important that no ELNES feature occurs within the energy range taken into consideration. ${ }^{14}$ During model fitting, $E_{\mathrm{m}}$ and $I_{\mathrm{m}}$ were constrained by the maximum counts and energy values of the experimental spectra. $E_{\mathrm{k}}$ was generally constrained in the region of $280-283 \mathrm{eV}$ (the starting point of the experimental spectrum). The parameter $r$ determines the shape of the model, and so, it was constrained such that the model obtains a shape, which matches that of the experimental spectrum. In initial literature reports, the ascending region of the experimental spectrum only was used within this model (289-291 eV was used for $\left.\mathrm{PET}^{14}\right)$. However, the shape of the carbon edge depends on the energy resolution, ${ }^{12}$ necessitating the inclusion of additional data points to reliably reproduce the shape of the edge. Due to the limitations imposed by energy resolution and the low signal-to-noise ratio, fine-structure features beyond $295 \mathrm{eV}$ could not be identified in our experimental spectra. Consequently, post-300 eV energies (typically $300-310 \mathrm{eV}$ ) were included for refinement of the model parameters. The fitted curve was subtracted from the experimental data (the carbon edge) to yield an ELNES signal, which could be decomposed into the contributing Gaussian peaks. Due to the large number of local minima, for chemically complex polymers that have several electron energy-loss peaks (a highly complex DOS), the peak energies were tightly constrained. For example, for the carbon edge of simple hydrocarbons such as PE and PP (Figure 1), for a given number of peaks (the lowest number to reasonably model the data), a wide energy window ( $5 \mathrm{eV}$ width) was able to be used, while for complex polymers such as PBT (Figure 2b), a number of peaks were specified and tight constraints $(1 \mathrm{eV}$ width max.) on the peak energies were required to obtain a representative fit. The constraints were tightened around the peak energies reported by XAS/NEXAFS. For comparison purposes, the energy scale of polymer NEXAFS data was shifted such that the peak due to the $\mathrm{C} 1 \mathrm{~s}-\pi^{*}{ }_{\mathrm{C}=\mathrm{C}}$ transition appears at $285 \mathrm{eV}$.

The processing was performed on individual datasets, and the results were averaged for each electron energy-loss peak observed. Subsequently, error is reported as the maximum deviation from the average. Due to the broad nature of $\sigma^{*}$ peaks, the error is usually high on the binding energy values obtained for $\sigma^{*}$ transitions from EELS data, though the relatively narrow $\sigma^{*}$ peak due to the $\mathrm{C}-\mathrm{H}$ transition is an exception. The $\sigma^{*} \mathrm{C}-\mathrm{H}$ transition often complicates the process of peak assignment in cases where peak overlap is expected due to the presence of heteroatoms.

\section{ASSOCIATED CONTENT}

\section{Supporting Information}

The Supporting Information is available free of charge at https://pubs.acs.org/doi/10.1021/acsomega.1c02939.

EELS chemical fingerprints of polystyrene (PS), polyethylene terephthalate (PET), and polymethylmethacrylate (PMMA); details of polymers used (PDF)

\section{AUTHOR INFORMATION}

\section{Corresponding Authors}

Alison M. Funston - School of Chemistry and ARC Centre of Excellence in Exciton Science, School of Chemistry, Monash University, Clayton, Victoria 3800, Australia; (1) orcid.org/ 0000-0002-4320-6434; Email: alison.funston@ monash.edu

Jayesh R. Bellare - Department of Chemical Engineering, IIT Bombay, Mumbai 400076, India; ㅇo이.org/0000-00026792-8327; Email: jb@iitb.ac.in

\section{Authors}

Ruchi Pal - IITB-Monash Research Academy, IIT Bombay, Mumbai 400076, India; Present Address: Present Address: Department of Materials Science and Engineering, IIT Delhi, Delhi 110016, India (R.P.); @ orcid.org/00000002-6769-9587

Laure Bourgeois - Monash Centre for Electron Microscopy and Department of Materials Science \& Engineering, Monash University, Clayton, Victoria 3800, Australia; (1) orcid.org/ 0000-0003-3961-5099

Matthew Weyland - Monash Centre for Electron Microscopy and Department of Materials Science \& Engineering, Monash University, Clayton, Victoria 3800, Australia; (1) orcid.org/ 0000-0003-1797-0268 
Arun K. Sikder - SABIC Research and Technology Pvt. Ltd., Bengaluru 562125, India; (1) orcid.org/0000-0001-90257093

Kei Saito - School of Chemistry, Monash University, Clayton, Victoria 3800, Australia; Present Address: Present Address: GSAIS, Kyoto University, 1 YoshidaNakaadachi-cho, Sakyo-ku, Kyoto 606-8306, Japan

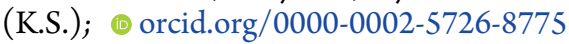

Complete contact information is available at: https://pubs.acs.org/10.1021/acsomega.1c02939

\section{Notes}

The authors declare no competing financial interest.

\section{ACKNOWLEDGMENTS}

This work was carried out within the ARC Centre of Excellence in Exciton Science, supported by the Australian Research Council (ARC) via grant CE170100026. The authors acknowledge the use of the instruments and scientific and technical assistance at the Monash Centre for Electron Microscopy, a Node of Microscopy Australia. This research used equipment funded by the Australian Research Council grant LE0454166. The authors also acknowledge IITBMonash Research Academy and Central facilities (CryoHRTEM), IIT Bombay, for their support and cooperation and SABIC for industrial sponsorship. R.P. thanks Asst. Prof. Tushar Gupta (National Institute of Technology Rourkela, India) for support in data processing and data representation within the manuscript. R.P. acknowledges the efforts of several people at IIT Bombay, Monash University and IIT Delhi, who helped in addressing reviewer comments. The authors thank all the reviewers, during this submission as well as the previous submissions, for constructive feedback to improve the manuscript.

\section{REFERENCES}

(1) Pal, R.; Sikder, A. K.; Saito, K.; Funston, A. M.; Bellare, J. R. Electron energy loss spectroscopy for polymers: a review. Polym. Chem. 2017, 8, 6927-6937.

(2) Egerton, R. F. Electron Energy-Loss Spectroscopy in the Electron Microscope; 3rd ed., Springer: New York, 2011.

(3) Pal, R.; Bourgeois, L.; Weyland, M.; Sikder, A. K.; Saito, K.; Funston, A. M.; Bellare, J. R. Chemical fingerprinting of polyvinyl acetate and polycarbonate using electron energy-loss spectroscopy. Polym. Chem. 2020, 11, 5484-5492.

(4) Dhez, O.; Ade, H.; Urquhart, S. G. Calibrated NEXAFS spectra of some common polymers. J. Electron Spectrosc. Relat. Phenom. 2003, $128,85-96$.

(5) Horiuchi, S.; Yin, D.; Ougizawa, T. Nanoscale Analysis of Polymer Interfaces by Energy-Filtering Transmission Electron Microscopy. Macromol. Chem. Phys. 2005, 206, 725-731.

(6) Ade, H.; Urquhart, S. G. NEXAFS spectroscopy and microscopy of natural and synthetic polymers. In Chemical applications of sunchrotron radiation; Sham, T. K., Ed.; World Scientific Publishing Co. Ltd.: Singapore, 2000.

(7) Kikuma, J.; Tonner, B. P. XANES spectra of a variety of widely used organic polymers at the C K-edge. J. Electron Spectrosc. Relat. Phenom. 1996, 82, 53-60.

(8) Wang, J.; Botton, G.; Hitchcock, A. P.; Dynes, J. J. Comparison of NEXAFS microscopy and TEM-EELS for studies of soft matter. Micron 2008, 39, 741-319.

(9) Egerton, R. F.; Lazar, S.; Libera, M. Delocalized radiation damage in polymers. Micron 2012, 43, 2-7.
(10) Leijten, Z. J. W. A.; Keizer, A. D. A.; De With, G.; Friedrich, H. Quantitative Analysis of Electron Beam Damage in Organic Thin Films. J. Phys. Chem. C 2017, 121, 10552-10561.

(11) Varlot, K.; Martin, J. M.; Quet, C. Physical and chemical changes in polystyrene during electron irradiation using EELS in the TEM: Contribution of the dielectric function. J. Microsc. 1998, 191, 187-194.

(12) Varlot, K.; Martin, J. M.; Quet, C. EELS analysis of PMMA at high spatial resolution. Micron 2001, 32, 371-378.

(13) Varlot, K.; Martin, J. M.; Gonbeau, D.; Quet, C. Chemical bonding analysis of electron-sensitive polymers by EELS. Polymer 1999, 40, 5691-5697.

(14) Varlot, K.; Martin, J. M.; Quet, C.; Kihn, Y. Towards subnanometer scale EELS analysis of polymers in the TEM. Ultramicroscopy 1997, 68, 123-133.

(15) Wang, C.; Duscher, G.; Paddison, S. J. Electron energy loss spectroscopy of polytetrafluoroethylene: experiment and first principles calculations. Microscopy 2014, 63, 73-83.

(16) Ditchfield, R. W.; Grubb, D. T.; Whelan, M. J. Electron energy loss studies of polymers during radiation damage. Philos. Mag. 1973, 27, 1267-1280.

(17) Ritsko, J. J. Electron energy loss spectroscopy of pristine and radiation damaged polyethylene. J. Chem. Phys. 1979, 70, 5343.

(18) Ohta, T.; Seki, K.; Yokoyama, T.; Morisada, I.; Edamatsu, K. Polarized XANES Studies of Oriented Polyethylene and Fluorinated Polyethylenes. Phys. Scr. 1990, 41, 150-153.

(19) Polvi, J. Low-Energy Radiation Effects in Polyethylene and Cellulose; 2013.

(20) Polvi, J.; Nordlund, K. Irradiation effects in high-density polyethylene. Nucl. Instrum. Methods Phys. Res., Sect. B 2013, 312, 5459.

(21) Imai, M.; Xu, L. G.; Ametani, K.; Tutiya, M. y-Irradiation of Polypropylene in Vacuum and in Air. J. Polym. Sci., Part A: Polym. Chem. 1989, 27, 1763-1773.

(22) Leung, B. O.; Hitchcock, A. P.; Brash, J. L.; Scholl, A.; Doran, A. Phase Segregation in Polystyrene-Polylactide Blends. Macromolecules 2009, 42, 1679-1684.

(23) Leung, B. O.; Hitchcock, A. P.; Cornelius, R.; Brash, J. L.; Scholl, A.; Doran, A. X-ray spectromicroscopy study of protein adsorption to a polystyrene-polylactide blend. Biomacromolecules 2009, 10, 1838-1845.

(24) Babanalbandi, A.; Hill, D. J. T.; O’Donnell, J. H.; Pomery, P. J.; Whittaker, A. An electron spin resonance study on y-irradiated poly (L-lactic acid) and poly (D,L-lactic acid). Polym. Degrad. Stab. 1995, 50, 297-304.

(25) Wady, P.; Wasilewski, A.; Brock, L.; Edge, R.; Baidak, A.; McBride, C.; Leay, L.; Griffiths, A.; Vallés, C. Effect of ionising radiation on the mechanical and structural properties of $3 \mathrm{D}$ printed plastics. Addit. Manuf. 2020, 31, 100907.

(26) Okajima, T.; Teramoto, K.; Mitsumoto, R.; Oji, H.; Yamamoto, Y.; Mori, I.; Ishii, H.; Ouchi, Y.; Seki, K. Polarized NEXAFS Spectroscopic Studies of Poly(butylene terephthalate), Poly(ethylene terephthalate), and Their Model Compounds. J. Phys. Chem. A 1998, 102, 7093-7099.

(27) Okajima, T.; Hara, K.; Yamamoto, M.; Seki, K. Near edge X-ray absorption fine structure spectroscopic and infrared reflection absorption spectroscopic studies of surface modification of poly(butylene terephthalate) induced by UV irradiation. Polymer 2012, 53, 2956-2963.

(28) Hitchcock, A. P.; Urquhart, S. G.; Rightor, E. G. Inner-shell spectroscopy of benzaldehyde, terephthalaldehyde, ethylbenzoate, terephthaloyl chloride and phosgene: models for core excitation of poly(ethylene terephthalate). J. Phys. Chem. 1992, 96, 8736-8750.

(29) Brydson, J. A. Plastics Materials; 7th Edition; Butterworth Heinemann: Oxford, 1999.

(30) Tourillon, G.; Garrett, R.; Lazarz, N.; Raynaud, M.; Reynaud, C.; Lécayon, G.; Viel, P. A NEXAFS study of thin polyacrylonitrile films electrochemically deposited on $\mathrm{Ni}$ : the effect of the film 
thickness and annealing treatment. J. Electrochem. Soc. 1990, 137, 2499-2501.

(31) Hill, D. J. T.; Lang, A. P.; O’Donnell, J. H.; Pomery, P. J. The effects of $\gamma$-radiation on copolymers of styrene and acrylonitrile. Polym. Degrad. Stab. 1992, 38, 205-218.

(32) Egerton, R. F.; Li, P.; Malac, M. Radiation damage in the TEM and SEM. Micron 2004, 35, 399-409.

(33) Tenailleau, H. Characterisation of Si-C-N-0 Ceramic Fibres through EFTEM; 1993. 